Poincare Journal of Analysis E Applications

Vol. 2014 (2), 71 - 75

CPoincare Publishers

\title{
ON BLOCK TRANSFINITE SEQUENCES IN BANACH SPACES
}

\author{
GHANSHYAM SINGH AND S.K. SHARMA ${ }^{\dagger}$
}

Date of Receiving : $\quad$ 20.10.2014

Date of Acceptance : $\quad$ 27. 12. 2014

\begin{abstract}
We introduced the concept of block transfinite sequences in Banach spaces. We prove that every block transfinite sequences with respect to a transfinite basis, more generally, a transfinite basic sequence (minimal transfinite sequence) is basic (respectively, minimal). The existence of a complete minimal transfinite sequence as a super transfinite sequence of block transfinite sequence with respect to a complete minimal transfinite sequence in a Banch space has been established. Finally, it is shown that every block transfinite sequence with respect to a shrinking minimal transfinite sequence is shrinking minimal.
\end{abstract}

\section{Introduction}

Bessaga [1] introduced the concept of monotone transfinite basis. Infact, he called it 'monotone basis of type $v^{\prime}$. Subsequently Bessaga [2] replaced the condition of monotonically by a weaker condition of uniform boundedness and introduced the term 'projection basis of type $v^{\prime}$. However, the definition of transfinite basis, we use in the present note is due to Dorembus [4]. Very recently, Jain And Ahmad [5] obtained an inequality characterizing transfinite basic sequences, analogous to the Nikol'skill inequality for bases. Further, Jain and Kaushik studied boundedly complete transfinite bases in [6].

In the present note, we introduced the concept of block transfinite sequences in Banach spaces. We prove that every block transfinite sequences with respect to a transfinite basis, more generally, a transfinite basic sequence (minimal transfinite sequence) is basic (respectively, minimal). The existence of a complete minimal transfinite sequence as a super transfinite sequence of block transfinite sequence with respect to a complete minimal transfinite sequence in a Banch space has been established. Finally, it is shown that every block transfinite sequence with respect to a shrinking minimal transfinite sequence is shrinking minimal.

2010 Mathematics Subject Classification. 42C15, 46B15.

Key words and phrases. Basis, Block Sequences.

Communicated by. Shashank Goel

${ }^{\dagger}$ Corresponding author. 